\title{
Analytical model for double split ring resonators with arbitrary ring width
}

\author{
Zhurbenko, Vitaliy; Jensen, Thomas; Krozer, Viktor; Meincke, Peter
}

Published in:

Microwave \& Optical Technology Letters

Link to article, DOI:

10.1002/mop.23104

Publication date:

2008

Document Version

Peer reviewed version

Link back to DTU Orbit

Citation $(A P A)$ :

Zhurbenko, V., Jensen, T., Krozer, V., \& Meincke, P. (2008). Analytical model for double split ring resonators with arbitrary ring width. Microwave \& Optical Technology Letters, 50(2), 511-515.

https://doi.org/10.1002/mop.23104

\section{General rights}

Copyright and moral rights for the publications made accessible in the public portal are retained by the authors and/or other copyright owners and it is a condition of accessing publications that users recognise and abide by the legal requirements associated with these rights.

- Users may download and print one copy of any publication from the public portal for the purpose of private study or research.

- You may not further distribute the material or use it for any profit-making activity or commercial gain

- You may freely distribute the URL identifying the publication in the public portal

If you believe that this document breaches copyright please contact us providing details, and we will remove access to the work immediately and investigate your claim 


\title{
Analytical Model for Double Split Ring Resonators with Arbitrary Ring Width
}

\author{
Vitaliy Zhurbenko, Thomas Jensen, Viktor Krozer, and Peter Meincke
}

Technical University of Denmark, Ørsted•DTU, ElectroScience, Ørsteds Plads, building 348, 2800

Kgs. Lyngby, Denmark, Phone:+4545253820, Fax:+4545931634, E-mail:vz@oersted.dtu.dk

\section{ABSTRACT}

For the first time, the analytical model for a double split ring resonator with unequal width rings is developed. The proposed models for the resonators with equal and unequal widths are based on an impedance matrix representation and provide the prediction of performance in a wide frequency range. A phase compensation is implemented to adjust for the difference in length of the two rings, resulting in an accurate calculation of the resonant frequencies.

\section{INDEX TERMS}

Split ring resonator, microstrip circuit, coupled lines, impedance matrix.

Artificial materials with periodic structures, generally denoted as metamaterials, with simultaneously negative permeability and permittivity have received considerable attention due to novel applications in physics and electromagnetics, such as reversal of Snell's law, microwave filters, and media with prescribed electromagnetic (EM) properties. Such materials can be realized using periodic inclusion of variously shaped metals into a host medium. The most prominent candidate for such structures has been the split-ring resonator. Such materials can exhibit effective permeability and permittivity properties beyond those occurring in naturally found materials. Artificial materials (metamaterials) are central to the research on negative permeability and permittivity, or left-handed materials. The key interest in these materials is the development of novel microwave and optical devices and materials based on left handedness and/or negative refractive index [1]-[3]. Double split ring resonators (SRR) are key components in these materials, also referred to as SRR particle [2], which exhibits only a resonant magnetic polarizability.

Microstrip double split ring resonators have recently attracted a great deal of interest for the design of compact microwave filters with superior characteristics [4]-[6]. The prediction of EM properties of such structures has been based on full-wave numerical simulations typically in 3-D, as demonstrated for example in [7] with considerable computational effort in terms of simulation time and memory storage. This has led to a number of attempts to develop analytical models using lumped circuit elements for the SRR particle [8]-[14]. These attempts are either limited in the resonance frequency prediction focusing only on a single resonance, or are based on solutions of differential circuit equations with parameters determined from EM simulations, which does not represent a breakthrough in the analysis of such structures. An accurate analytical model for the SRR's has the potential to drastically lower the design time and will make high performance microwave filters based on SRR's a practical option in many commercial applications. The model presented in [12], [13] provides a wideband analysis, but, unfortunately, doesn't take into account the presence of a ground plane in planar structures.

To the knowledge of the authors an analytical model for unequal width SRR's has not been presented previously in the open literature. In this letter we present an accurate yet simple analytical model for double SRR based on an impedance matrix representation for coupled transmission lines. We focus here on double 
SRR with a ground plane, which leads to the utilization of microstrip components. The parameters of the coupled line sections are related to the physical characteristics of the transmission line technology, making it possible to directly convert even and odd mode impedances into transmission line widths and gaps, something that is not possible with the lumped element circuit models. The general analytical model is derived below for double SRR with unequal width rings, which represents a generalization of the standard SRR. Expressions are provided which extend the proposed model to equal width case and the model accuracy is discussed comparing different simulators with proven accuracy with the compact model.

The SRR structure, originally proposed by Pendry et al. [15], consists of a pair of equal width concentric rings with gaps etched on opposite sides. The SRR's call for a fine mesh density to accurately map the circular geometry, the gaps, and the coupling between the rings, and results in a large number of unknowns with excessive computational time. Typical computational times for a 3-D EM simulation using CST Microwave Studio $2006 b^{1}$ are in excess of $12 \mathrm{hr}$ on a $2.7 \mathrm{GHz}$ Pentium machine with 2.5 GB RAM memory and $6 \cdot 10^{6}$ mesh cells.

The configuration considered in this letter consists of two unequal width split rings, as shown in Fig. 1(a). The proposed model representation for the unequal width double SRR is shown in Fig.1(b). The model is based on two coupled line sections with a length equal to

$$
l_{c l}=\pi\left(r+\frac{w_{1}}{2}\right)-g
$$

This length corresponds to the average length of the inner semiring. The coupled line sections are a compact representation of the model in [12], [13]. The coupled line sections are described in terms of even and odd mode impedance and propagation parameters, which are available for a large range of different realizations using standard microwave theory.

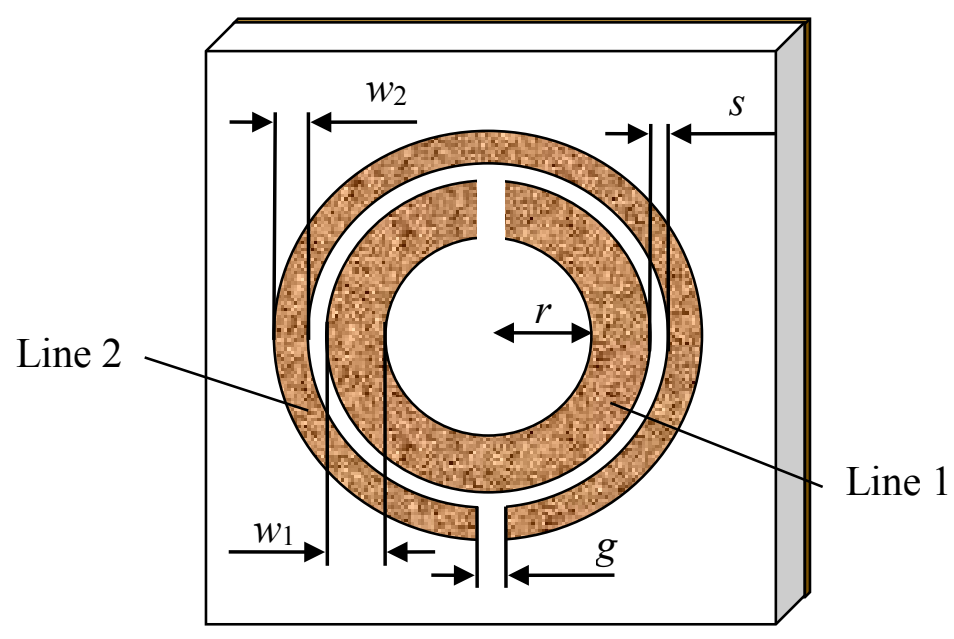

(a)

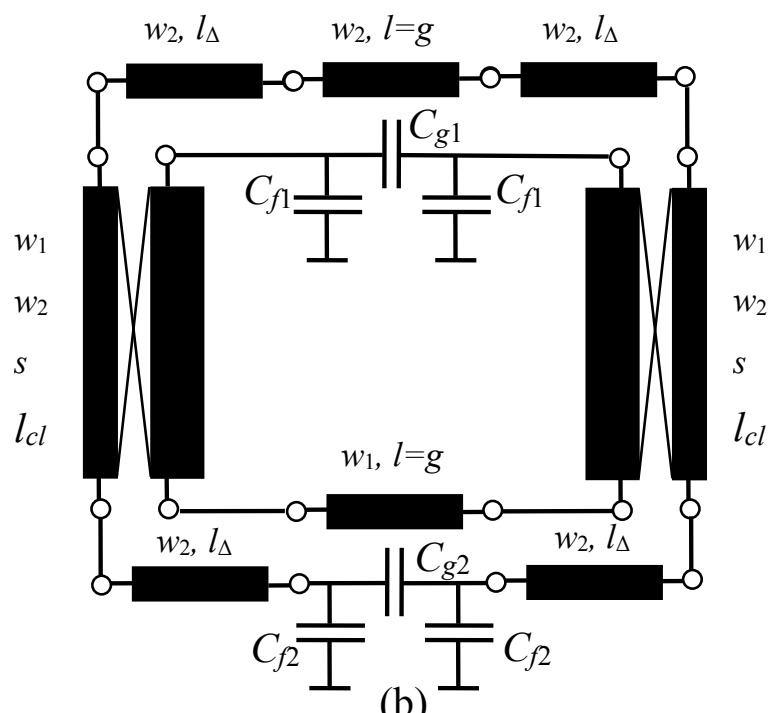

(b)

Fig. 1. Microstrip double SRR geometry (a) and corresponding model representation (b).

It is obvious that the geometrical lengths of the two resonators are not equal. In most practical cases this difference between electrical lengths at first resonance frequency reaches a few tens of degrees and even higher. Preliminary model investigations showed that it is not sufficient to describe double SRR by just two

${ }^{1}$ CST Microwave Studio version 2006b is a trademark of CST GmbH. 
coupled line sections with a length equal to the average resonator lengths. To compensate for this difference four transmission lines with a total length equal to

$$
4 l_{\Delta}=2\left(\pi\left(r+w_{1}+\frac{w_{2}}{2}+s\right)-g-l_{c l}\right)
$$

have to be introduced, as shown in Fig. 1(b). Such a phase compensation significantly improves the model accuracy resulting in good agreement between simulated and calculated results, as shown later.

An equivalent circuit for the gap $g$ of the resonators is a symmetric 2-port $\pi$-network, as shown in Fig. 1(b). The capacitance $C_{g 1,2}$ is due to the charge buildup between the two line contacts. The capacitance $C_{f 1,2}$ is due to the fringing fields at the open line ends. The calculation of values for these capacitors in case of microstrip lines is based on well established empirical expressions given in literature [16]-[18] and is not considered here. A transmission line with a length equal to $g$ unites the two semirings. The semi rings are represented by coupled line sections, to form a ring.

The circuit in Fig. 1(b) is transformed to an impedance matrix network with the corresponding matrix representation of the circuit elements. This network is shown in Fig. 2.

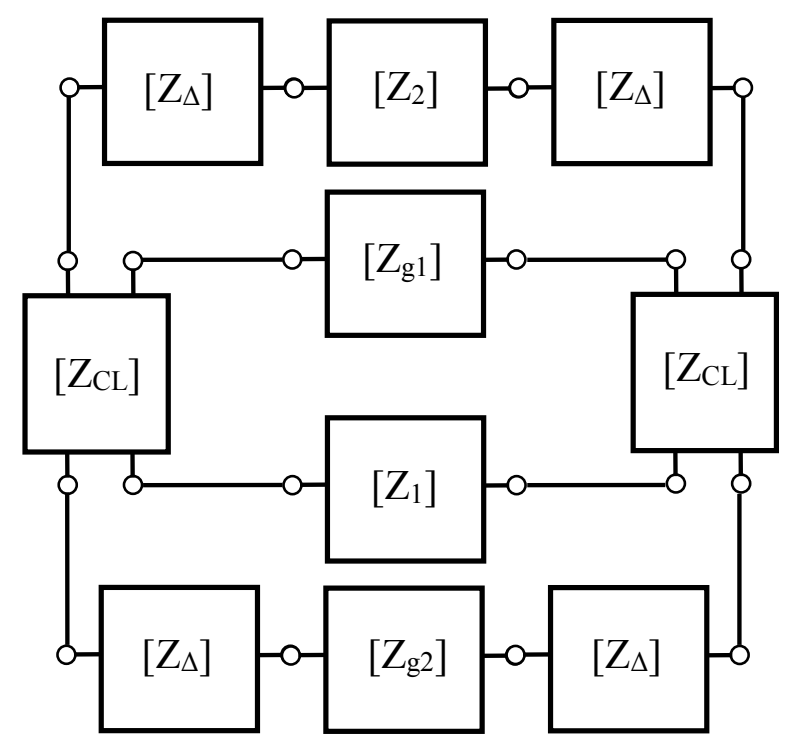

Fig. 2. Double SRR model based on an impedance matrix representation.

The various impedance matrices in Fig. 2 are described below.

$\left[Z_{\mathrm{CL}}\right]$ is the impedance matrix for the coupled lines 4-port representation. A total of six quantities is required to describe the asymmetric coupled lines [18]: $Z_{c l}$ and $Z_{\pi l}$, which are, respectively, the characteristic impedances of line 1 for $c$ and $\pi$ modes of propagation; $\gamma_{c}$ and $\gamma_{\pi}$, the propagation constants of $c$ and $\pi$ modes; $R_{c}$ and $R_{\pi}$, the ratios of the voltages on the two lines for $c$ and $\pi$ modes. The elements of the impedance matrix $\left[Z_{C L}\right]$ are given by

$$
Z_{11}=Z_{44}=\frac{Z_{c 1} \operatorname{coth}\left(\gamma_{c} l_{c l}\right)}{1-R_{c} / R_{\pi}}+\frac{Z_{\pi 1} \operatorname{coth}\left(\gamma_{\pi} l_{c l}\right)}{1-R_{\pi} / R_{c}},
$$




$$
\begin{gathered}
Z_{22}=Z_{33}=\frac{Z_{c 1} R_{c}^{2} \operatorname{coth}\left(\gamma_{c} l_{c l}\right)}{1-R_{c} / R_{\pi}}+\frac{Z_{\pi 1} R_{\pi}^{2} \operatorname{coth}\left(\gamma_{\pi} l_{c l}\right)}{1-R_{\pi} / R_{c}}, \\
Z_{12}=Z_{21}=Z_{34}=Z_{43}=\frac{Z_{c 1} R_{c} \operatorname{coth}\left(\gamma_{c} l_{c l}\right)}{1-R_{c} / R_{\pi}}+\frac{Z_{\pi 1} R_{\pi} \operatorname{coth}\left(\gamma_{\pi} l_{c l}\right)}{1-R_{\pi} / R_{c}}, \\
Z_{13}=Z_{31}=Z_{24}=Z_{42}=\frac{Z_{c 1} R_{c} \operatorname{csch}\left(\gamma_{c} l_{c l}\right)}{\left(1-R_{c} / R_{\pi}\right)}+\frac{Z_{\pi 1} R_{\pi} \operatorname{csch}\left(\gamma_{\pi} l_{c l}\right)}{\left(1-R_{\pi} / R_{c}\right)}, \\
Z_{14}=Z_{41}=\frac{Z_{c 1} \operatorname{sch}\left(\gamma_{c} l_{c l}\right)}{\left(1-R_{c} / R_{\pi}\right)}+\frac{Z_{\pi 1} \operatorname{csch}\left(\gamma_{\pi} l_{c l}\right)}{\left(1-R_{\pi} / R_{c}\right)}, \\
Z_{23}=Z_{32}=\frac{Z_{c 1} R_{c}^{2} \operatorname{csch}\left(\gamma_{c} l_{c l}\right)}{\left(1-R_{c} / R_{\pi}\right)}+\frac{Z_{\pi 1} R_{\pi}^{2} \operatorname{csch}\left(\gamma_{\pi} l_{c l}\right)}{\left(1-R_{\pi} / R_{c}\right)},
\end{gathered}
$$

where $l_{c l}$ is the length of the coupled line section, as shown in Fig. 1(b).

The matrices for the transmission lines are given by

$$
\left[Z_{i}\right]=\left[\begin{array}{ll}
Z_{0(i)} \operatorname{coth}\left(\gamma_{i} g\right) & Z_{0(i)} \operatorname{csch}\left(\gamma_{i} g\right) \\
Z_{0(i)} \operatorname{csch}\left(\gamma_{i} g\right) & Z_{0(i)} \operatorname{coth}\left(\gamma_{i} g\right)
\end{array}\right], \quad\left[Z_{\Delta}\right]=\left[\begin{array}{ll}
Z_{0(2)} \operatorname{coth}\left(\gamma_{2} l_{\Delta}\right) & Z_{0(2)} \operatorname{csch}\left(\gamma_{2} l_{\Delta}\right) \\
Z_{0(2)} \operatorname{csch}\left(\gamma_{2} l_{\Delta}\right) & Z_{0(2)} \operatorname{coth}\left(\gamma_{2} l_{\Delta}\right)
\end{array}\right] .
$$

Here $Z_{0(i)}$ is the characteristic impedance and $\gamma_{i}$ is the propagation constant of line $i$, with $i=1,2$, respectively. The calculation of the electrical parameters for the microstrip lines and coupled lines can be performed using empirical expressions [18] or available transmission line calculators.

The impedance matrix for the microstrip gap is

$$
\left[Z_{g i}\right]=\left[\begin{array}{cc}
\frac{\mathrm{C}_{f i}+\mathrm{C}_{g i}}{j \omega \mathrm{C}_{f i}\left(2 \mathrm{C}_{g i}+\mathrm{C}_{f i}\right)} & \frac{\mathrm{C}_{g i}}{j \omega \mathrm{C}_{f i}\left(2 \mathrm{C}_{g i}+\mathrm{C}_{f i}\right)} \\
\frac{\mathrm{C}_{g i}}{j \omega \mathrm{C}_{f i}\left(2 \mathrm{C}_{g i}+\mathrm{C}_{f i}\right)} & \frac{\mathrm{C}_{f i}+\mathrm{C}_{g i}}{j \omega \mathrm{C}_{f i}\left(2 \mathrm{C}_{g i}+\mathrm{C}_{f i}\right)}
\end{array}\right] .
$$

Using the model representation shown in Fig. 2 and relations (3)-(5) the response of the resonator can be predicted in a wide frequency range.

Since an equal width double SRR is a special case of an unequal width double SRR the given expressions can also be used for the equal width case, by setting $w_{1}=w_{2}=w$. This leads to implementation of impedance matrix for symmetric coupled lines in the model shown in Fig. 2.

For the symmetric coupled lines in a nonhomogeneous medium the electrical parameters become: $R_{c}=1$, $R_{\pi}=-1$, and $Z_{c 1}=Z_{0 e}$, the even-mode impedance; $Z_{\pi 1}=Z_{0 o}$, the odd-mode impedance, with $\gamma_{c, \pi}=\gamma_{e, o}$, the even- and odd-mode propagation constants. These parameters are substituted in (3) to obtain an analytical model for the equal width double SRR. 
The accuracy of the proposed model has been validated using a planar SRR in a microstrip configuration, because accurate models exist for all elements of the proposed model. SRR excitation at the gap and with a line have been studied. The first 4 resonances have been calculated using the proposed model and full-wave EM solvers based on method of moments (Agilent MOMENTUM) and a FITD (CST Microwave Studio). The considered configuration is based on a substrate with a dielectric constant $\varepsilon_{\mathrm{r}}=3.38$ and thickness $\mathrm{h}=0.8 \mathrm{~mm}$. The frequency range for the MoM simulation is restricted to the first four resonances to reduce the computational load.

The typical wideband response of SRR's is presented in Fig. 3.

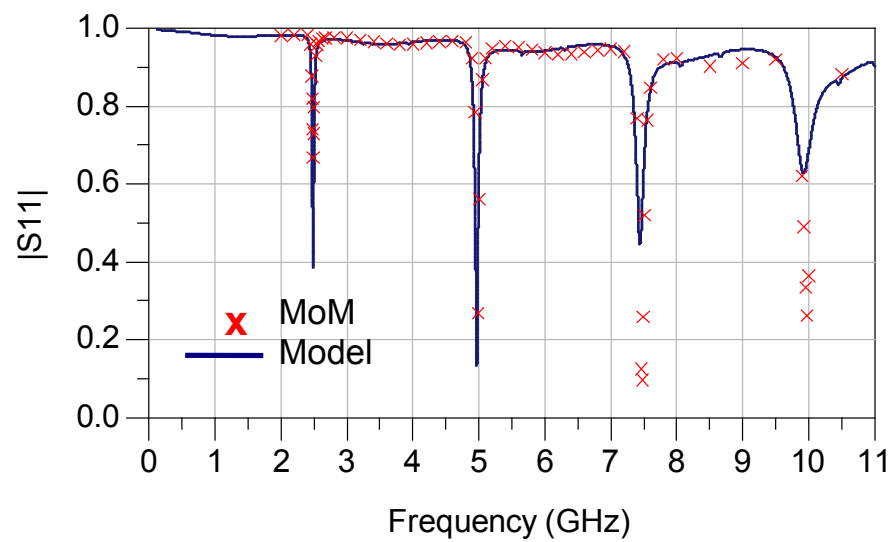

(a)

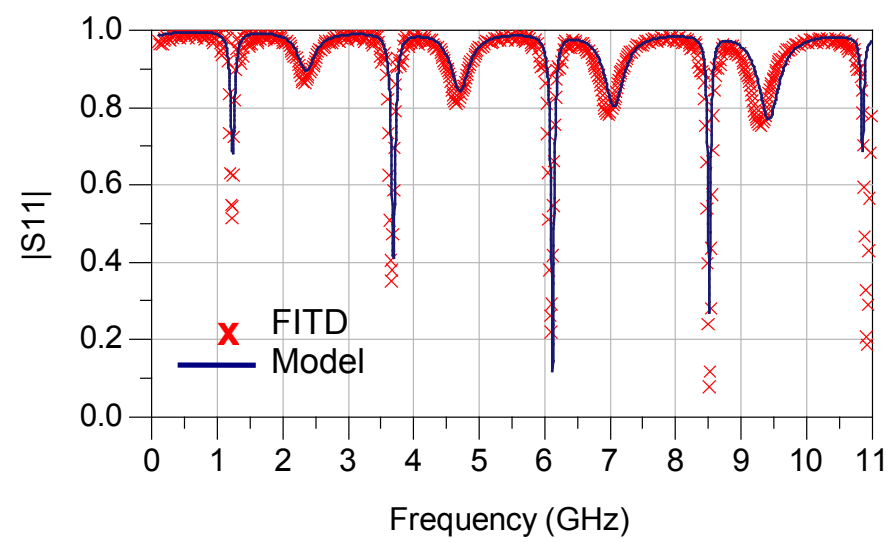

(b)

Fig. 3: Comparison between full-wave EM simulations and the proposed model for microstrip SRR's with geometry given in Table 1. (a) Equal width SRR with transmission line excitation. (b) Unequal width SRR with excitation at the gap.

A comparison between the presented model and full-wave electromagnetic simulations shows excellent agreement. As can be seen in Fig. 3, the resonant frequencies are accurately predicted in a wide frequency range by the model and the EM simulators. The SRR parameters and the difference between the calculated and simulated first, second, third and fourth resonant frequencies $\delta f_{1,2,3,4}$ are given in Table 1 for equal width and unequal width SRR.

TABLE 1: SRR GEOEMTRICAL PARAMETERS AND COMPARISON OF PREDICTED RESONANCE FREQUENCIES

EQUAL WIDTH MICROSTRIP SRR

\begin{tabular}{l|c|c|c|c|c|c|c|c}
\hline \hline Parameter & $\begin{array}{c}r \\
(\mathrm{~mm})\end{array}$ & $\begin{array}{c}w \\
(\mathrm{~mm})\end{array}$ & $\begin{array}{c}s \\
(\mathrm{~mm})\end{array}$ & $\begin{array}{c}g \\
(\mathrm{~mm})\end{array}$ & $\begin{array}{c}\delta f_{1} \\
(\%)\end{array}$ & $\begin{array}{c}\delta f_{2} \\
(\%)\end{array}$ & $\begin{array}{c}\delta f_{3} \\
(\%)\end{array}$ & $\begin{array}{c}\delta f_{4} \\
(\%)\end{array}$ \\
\hline Value & 12.7 & 0.2 & 0.17 & 0.17 & 0.02 & 0.06 & 0.17 & 0.3 \\
\hline \hline
\end{tabular}

UNEQUAL WIDTH MICROSTRIP SRR

\begin{tabular}{l|c|c|c|c|c|c|c|c|c}
\hline \hline Parameter & $\begin{array}{c}r \\
(\mathrm{~mm})\end{array}$ & $\begin{array}{c}w_{1} \\
(\mathrm{~mm})\end{array}$ & $\begin{array}{c}w_{2} \\
(\mathrm{~mm})\end{array}$ & $\begin{array}{c}S \\
(\mathrm{~mm})\end{array}$ & $\begin{array}{c}g \\
(\mathrm{~mm})\end{array}$ & $\begin{array}{c}\delta f_{1} \\
(\%)\end{array}$ & $\begin{array}{c}\delta f_{2} \\
(\%)\end{array}$ & $\begin{array}{c}\delta f_{3} \\
(\%)\end{array}$ & $\begin{array}{c}\delta f_{4} \\
(\%)\end{array}$ \\
\hline Value & 12.5 & 0.4 & 0.2 & 0.17 & 0.17 & 0.81 & 0.71 & 1.01 & 0.66 \\
\hline \hline
\end{tabular}

The first resonant frequency is predicted with an accuracy better than $0.02 \%$ for the equal width case. The differences in accuracy between the equal width and unequal width cases are mainly caused by the varying accuracy of the relations used for parameters of symmetric and asymmetric coupled lines in the 
model. These relations are well developed for symmetric coupled lines, which makes the accurate modeling of equal width double SRR possible, but are less studied for the unequal width case. Although the obtained accuracy for the unequal width case is lower it is deemed sufficient for most applications.

In conclusion we have presented, for the first time, an analytical circuit based model for the unequal width double SRR. It has been shown that the characteristics of double SRR's may be accurately described using an impedance matrix representation for coupled transmission lines. The model has been verified for the relevant special case of a microstrip realization of the SRR. Electromagnetic simulation using different full-wave methods in 2D and 3D are used as a reference for the developed model validation. An excellent agreement between simulated and proposed model results is achieved in prediction of higher order resonances. The proposed analytical model enables a simple and efficient design of double SRR structures for various applications in a wide frequency range. Especially, microwave filter design utilizing SRR is greatly facilitated using this model. As the next step, meta-material design with negative permeability will be demonstrated in future contributions.

\section{REFERENCES}

[1] F. Martín, J. Bonache, F. Falcone, And M. Sorolla, R. Marqués: "Split Ring Resonator-Based LeftHanded Coplanar Waveguide", Appl. Phys. Lett., Vol. 83, No. 22, pp. 2003.

[2] C. R. Simovski and B. Sauviac, "Towards creating isotropic microwave composites with negative refraction”, Radio Science, vol. 39, pp. RS2014.1-18, 2004

[3] T. Decoopman, O. Vanbésien, and D. Lippens, "Demonstration of a Backward Wave in a Single Split Ring Resonator and Wire Loaded Finline", IEEE Microwave And Wireless Comp. Lett., Vol. 14, No. 11, pp. 507-9, 2004.

[4] J. García-García, et al., "Miniaturized Microstrip and CPW Filters Using Coupled Metamaterial Resonators," IEEE Transactions on Microwave Theory and Techniques, vol.54, no. 6, pp. 2628-2634, June 2006.

[5] Gil, et al.," Metamaterials in Microstrip Technology for Filter Applications" 2005 IEEE Antennas and Propagation Society International Symposium, vol. 1A, pp. 668-671, July 2005.

[6] J. García-García, et al., "Microwave Filters With Improved Stopband Based on Sub-Wavelength Resonators," IEEE Transactions on Microwave Theory and Techniques, vol.53, no. 6, pp. 1997-2006, June 2005.

[7] T. Weiland et al, "Ab initio numerical simulation of left-handed metamaterials: Comparison of calculations and experiments”, J. Appl. Phys., Vol. 90, No. 10, Nov. 2001.

[8] J. D. Baena, et al., "Equivalent-Circuit Models for Split-Ring Resonators and Complementary SplitRing Resonators Coupled to Planar Transmission Lines," IEEE Transactions on Microwave Theory and Techniques, Vol. 53, No. 4, April 2005.

[9] B. Sauviac, C. R. Simovski, S. A. Tretyakov, "Double Split-Ring Resonators: Analytical Modeling and Numerical Simulations”, Electromagnetics, Vol. 24, pp. 317-338, 2004.

[10] J. Martel, et al, “A New LC Series Element for Compact Bandpass Filter Design”, IEEE Microwave \& Wireless Components Letters, Vol. 14, No. 5, pp. 210-2, May 2004.

[11] B. K. Kanaujia and B. R. Vishvakarma, "Analysis Of Two-Concentric Annular Ring Microstrip Antenna”, Microwave And Optical Technology Letters, Vol. 36, No. 2, pp. 104-8, Jan. 2003. 
[12] M. Shamonin, E. Shamonina, V. Kalinin, and L. Solymar, "Properties of a metamaterial element: Analytical solutions and numerical simulations for a singly split double ring “, J. Appl. Phys., Vol. 95, No. 7, pp. 3778-3784, April 2004.

[13] M. Shamonin, E. Shamonina, V. Kalinin, and L. Solymar, "Resonant Frequencies Of A Split-Ring Resonator: Analytical Solutions And Numerical Simulations," Microwave And Optical Technology Letters, Vol. 44, No. 2, pp. 133-136, January 202005.

[14] S. Hrabar, J. Bartolic, "Simplified Analysis of Split-Ring Resonator Used in Backward MetaMaterial”. Proc. MMET, 2002.

[15] Pendry, J. B., A. J. Holden, D. J. Robbins, and W. J. Stewart, "Magnetism from conductors and enhanced nonlinear phenomena," IEEE Transactions on Microwave Theory and Techniques, vol.47, pp. 2075-2084, 1999.

[16] M. Kirschning, R. H. Jansen, N. H. L. Koster, "Measurement And Computer-Aided Modelling Of Microstrip Discontinuities By An Improved Resonator Method," IEEE MTT-S International Microwave Symposium Digest, pp. 495-497, 1983.

[17] E. Hammerstad, "Computer-Aided Design Of Microstrip Couplers With Accurate Discontinuity Models,” IEEE MTT-S International Microwave Symposium Digest, pp. 54-56, 1981.

[18] N. H. L. Koster, R. H. Jansen, "The Equivalent Circuit of the Asymmetrical Series Gap in Microstrip and Suspended Substrate Lines," Transactions on Microwave Theory and Techniques, vol. MTT 30, no. 8, August 1982.

[19] K. Tripathi, "Asymmetric Coupled Transmission Lines in an Inhomogeneous Medium," IEEE Trans. Microwave Theory \& Tech., vol. 23, no. 9, pp. 734-739, September 1975.

[20] R. Mongia, I. Bahl, P. Bhartia, RF and microwave coupled line circuits. Norwood: Artech House microwave library, 1999. 